\title{
A New Latent Cure Rate Marker Model for Survival Data: Supplementary Material
}

Sungduk Kim, Yingmei Xi, and Ming-Hui Chen*

February 10, 2009

\section{Checking the Proportional Hazards (PH) Assump- tion}

To examine the proportional hazards (PH) assumption for the cumulative hazard function $H(y)$ for non-cured subjects in Equation (5), we consider the generalized odds-rate hazards $(\mathrm{GORH})$ model discussed in Banerjee et al. (2007). Under the GORH model for $H(y)$, we have

$$
S_{i}\left(y \mid \boldsymbol{x}_{i}, \boldsymbol{\beta}, \theta_{g_{i}}, \xi\right)=\exp \left(-\theta_{g_{i}}\left[1-\left\{\frac{1}{1+\xi \exp \left(\boldsymbol{x}_{i}^{\prime} \boldsymbol{\beta}\right) H_{0}(y)}\right\}^{\frac{1}{\xi}}\right]\right) .
$$

It is easy to show that $\lim _{\xi \rightarrow 0} S_{i}\left(y \mid \boldsymbol{x}_{i}, \boldsymbol{\beta}, \theta_{g_{i}}, \xi\right)=S_{i}\left(y \mid \boldsymbol{x}_{i}, \boldsymbol{\beta}, \theta_{g_{i}}\right)$, where $S_{i}\left(y \mid \boldsymbol{x}_{i}, \boldsymbol{\beta}, \theta_{g_{i}}\right)$ is defined in Equation (5). Thus, the PH model for $F(y)$ is a special case of the GORH model when $\xi \rightarrow 0$. When $\xi=1$, the GORH model reduces to the proportional odds model. We computed LPMLs and DICs for various choices of $\xi, J$, and $G$ for the LCRM with the GORH model for $H(y)$. For the LCRM with $G=3$ and $J=5$, LPMLs and DICs are -817.3 and 1620.0 for $\xi=1,-817.0$ and 1616.1 for $\xi=0.5$, and -816.1 and 1614.9 for $\xi=0.1$. For the LCRM with $G=3$, LPMLs and DICs are -824.8 and 1629.7 for $J=1$ and -821.7 and 1629.5 for $J=10$ for $\xi=1$; -924.1 and 1627.8 for $J=1$ and

\footnotetext{
*Corresponding Author: Ming-Hui Chen, Department of Statistics, University of Connecticut, 215
} Glenbrook Road, U-4120, Storrs, CT 06269, USA (E-mail: mhchen@stat.uconn.edu). 
-820.6 and 1626.9 for $J=10$ for $\xi=0.5$; and -823.1 and 1626.7 for $J=1$ and -819.8 and 1625.1 for $J=10$ for $\xi=0.1$. Comparing these results to those under the PH model for $H(y)$, we see that the LCRM with the PH model for $H(y)$ fits the data better than the one with the GORH model. The fit improves when $\xi$ gets smaller and the LPMLs and DICs under the GORH model with $\xi=0.1$ are very close to those under the PH model. The LPMLs and DICs were also computed for other values of $G$ and the similar patterns were observed. These results empirically confirm that the $\mathrm{PH}$ assumption for $H(y)$ may be appropriate for this data set.

\section{Computational Development}

We first consider a fixed $G$. To sample from the posterior distribution given in Equation (14), we propose a two-step MCMC sampling algorithm, which consists of Step 1: Parent MCMC and Step 2: Multigrid Monte Carlo (MGMC) Adjustment. In the Parent MCMC step, we require sampling from the conditional distributions: (i) $\left[\boldsymbol{\lambda} \mid \boldsymbol{\beta}, \boldsymbol{\theta}, \boldsymbol{\phi}, \boldsymbol{N}, \boldsymbol{g}, D_{\text {obs }}\right]$ and (ii) $\left[\boldsymbol{\beta}, \boldsymbol{\theta}, \boldsymbol{\phi}, \boldsymbol{N}, \boldsymbol{g} \mid \boldsymbol{\lambda}, D_{\text {obs }}\right]$. We briefly discuss how to sample from each of the above conditional posterior distributions. For (i),

$$
\begin{aligned}
& \lambda_{j} \mid \boldsymbol{\beta}, \boldsymbol{\theta}, \boldsymbol{\phi}, \boldsymbol{N}, \boldsymbol{g}, D_{o b s} \\
\sim & \operatorname{Gamma}\left(a_{0}+\sum_{i=1}^{n} \nu_{i} \delta_{i j}, b_{0}+\sum_{i=1}^{n} N_{i} \exp \left(\boldsymbol{x}_{i}^{\prime} \boldsymbol{\beta}\right)\left\{\delta_{i j}\left(y_{i}-s_{j-1}\right)+\sum_{k=j+1}^{J} \delta_{i k}\left(s_{j}-s_{j-1}\right)\right\}\right)
\end{aligned}
$$

for $j=1,2, \ldots, J$. Thus, sampling from the conditional posterior distributions of $\boldsymbol{\lambda}$ is straightforward. For (ii), we apply the collapsed Gibbs technique of Liu (1994) via the following identity:

$$
\left[\boldsymbol{\beta}, \boldsymbol{\theta}, \boldsymbol{\phi}, \boldsymbol{N}, \boldsymbol{g} \mid \boldsymbol{\lambda}, D_{o b s}\right]=\left[\boldsymbol{N} \mid \boldsymbol{\beta}, \boldsymbol{\theta}, \boldsymbol{\phi}, \boldsymbol{g}, \boldsymbol{\lambda}, D_{o b s}\right]\left[\boldsymbol{\beta}, \boldsymbol{\theta}, \boldsymbol{\phi}, \boldsymbol{g} \mid \boldsymbol{\lambda}, D_{o b s}\right]
$$

That is, we sample $\boldsymbol{\beta}, \boldsymbol{\theta}, \boldsymbol{\phi}$, and $\boldsymbol{g}$ after collapsing out $\boldsymbol{N}$. Observe that

$$
N_{i} \mid \boldsymbol{\beta}, \boldsymbol{\theta}, \boldsymbol{\phi}, \boldsymbol{g}, \boldsymbol{\lambda}, D_{\text {obs }} \sim \text { Poisson }\left(\theta_{g_{i}} \exp \left\{-\exp \left(\boldsymbol{x}_{i}^{\prime} \boldsymbol{\beta}\right) H_{0}^{*}\left(y_{i}\right)\right\}\right)+\nu_{i}
$$


for $i=1,2, \ldots, n$. Thus, sampling from the conditional posterior distributions of $\boldsymbol{N}$ is straightforward. Given $\boldsymbol{\theta}, \boldsymbol{\phi}, \boldsymbol{g}, \boldsymbol{\lambda}$, and $D_{o b s}$, the conditional posterior density for $\left[\boldsymbol{\beta} \mid \boldsymbol{\theta}, \boldsymbol{\phi}, \boldsymbol{g}, \boldsymbol{\lambda}, D_{o b s}\right]$ has the form

$$
\begin{aligned}
& \pi\left(\boldsymbol{\beta} \mid \boldsymbol{\theta}, \boldsymbol{\phi}, \boldsymbol{g}, \boldsymbol{\lambda}, D_{o b s}\right) \\
\propto & \prod_{i=1}^{n} \exp \left[\nu_{i}\left\{\boldsymbol{x}_{i}^{\prime} \boldsymbol{\beta}-\exp \left(\boldsymbol{x}_{i}^{\prime} \boldsymbol{\beta}\right) H_{0}^{*}\left(y_{i}\right)\right\}+\theta_{g_{i}} \exp \left\{-\exp \left(\boldsymbol{x}_{i}^{\prime} \boldsymbol{\beta}\right) H_{0}^{*}\left(y_{i}\right)\right\}\right] \pi(\boldsymbol{\beta}) .
\end{aligned}
$$

Therefore, we use the Metropolis-Hastings algorithm (Hastings, 1970) to sample $\boldsymbol{\beta}$ from (S.1). Also given $\boldsymbol{\beta}, \boldsymbol{\phi}, \boldsymbol{g}, \boldsymbol{\lambda}$, and $D_{o b s}$, the conditional posterior density of $\theta$ has the form

$$
\pi\left(\boldsymbol{\theta} \mid \boldsymbol{\beta}, \boldsymbol{\phi}, \boldsymbol{g}, \boldsymbol{\lambda}, D_{\text {obs }}\right) \propto \prod_{l=1}^{G} \theta_{l}^{A_{l}-1} \exp \left(-B_{l} \theta_{l}\right) 1\left(\theta_{1} \leq \cdots \leq \theta_{G}\right),
$$

where $A_{l}=a_{l}+\sum_{g_{i}=l} \nu_{i}, B_{l}=b_{l}+\sum_{g_{i}=l}\left[1-\exp \left\{-\exp \left(\boldsymbol{x}_{i}^{\prime} \boldsymbol{\beta}\right) H_{0}^{*}\left(y_{i}\right)\right\}\right]$. We use the truncated Gamma distribution to sample $\boldsymbol{\theta}$ from (S.2). The conditional posterior density for $\left[\boldsymbol{\phi} \mid \boldsymbol{\beta}, \boldsymbol{\theta}, \boldsymbol{g}, \boldsymbol{\lambda}, D_{\text {obs }}\right]$ has the form

$$
\pi\left(\boldsymbol{\phi}_{l} \mid \boldsymbol{\beta}, \boldsymbol{\theta}, \boldsymbol{g}, \boldsymbol{\lambda}, D_{o b s}\right) \propto \prod_{g_{i}=l} \frac{\exp \left(\boldsymbol{z}_{i}^{\prime} \boldsymbol{\phi}_{l}\right)}{\sum_{k=1}^{G} \exp \left(\boldsymbol{z}_{i}^{\prime} \boldsymbol{\phi}_{k}\right)} \times \pi\left(\boldsymbol{\phi}_{l}\right) .
$$

It can be shown that $\pi\left(\boldsymbol{\phi}_{l} \mid \boldsymbol{\beta}, \boldsymbol{\theta}, \boldsymbol{g}, \boldsymbol{\lambda}, D_{\text {obs }}\right)$ is log-concave in each component of $\boldsymbol{\phi}_{l}$. Therefore, we can use the adaptive rejection algorithm of Gilks and Wild (1992) to sample from $\left[\boldsymbol{\phi}_{l} \mid \boldsymbol{\beta}, \boldsymbol{\theta}, \boldsymbol{g}, \boldsymbol{\lambda}, D_{o b s}\right]$. Finally, given $\boldsymbol{\beta}, \boldsymbol{\theta}, \boldsymbol{\phi}, \boldsymbol{\lambda}$, and $D_{o b s}$, the conditional posterior density for $\left[\boldsymbol{g} \mid \boldsymbol{\beta}, \boldsymbol{\theta}, \boldsymbol{\phi}, \boldsymbol{\lambda}, D_{\text {obs }}\right]$ has the form

$$
\begin{aligned}
& \pi\left(g_{i} \mid \boldsymbol{\beta}, \boldsymbol{\theta}, \boldsymbol{\phi}, \boldsymbol{\lambda}, D_{\text {obs }}\right) \\
\propto & \exp \left(\nu_{i} \log \theta_{g_{i}}-\theta_{g_{i}}\left[1-\exp \left\{-\exp \left(\boldsymbol{x}_{i}^{\prime} \boldsymbol{\beta}\right) H_{0}^{*}\left(y_{i}\right)\right\}\right]\right) \exp \left(\boldsymbol{z}_{i}^{\prime} \boldsymbol{\phi}_{g_{i}}\right) .
\end{aligned}
$$

To sample $g_{i}$ from (S.4), we use the inverse transformation method for $\pi\left(g_{i} \mid \boldsymbol{\beta}, \boldsymbol{\theta}, \boldsymbol{\phi}, \boldsymbol{\lambda}, D_{\text {obs }}\right)$.

In the MGMC adjustment step, we use the MGMC scheme of Liu and Sabatti (2000). First, we integrate out $\boldsymbol{N}$ from the joint posterior distribution of $(\boldsymbol{\beta}, \boldsymbol{\theta}, \boldsymbol{\phi}, \boldsymbol{\lambda}, \boldsymbol{N}, \boldsymbol{g})$. Second, we take the group transformation $\zeta(\boldsymbol{\beta}, \boldsymbol{\theta}, \boldsymbol{\phi})=(\zeta \boldsymbol{\beta}, \zeta \boldsymbol{\theta}, \zeta \boldsymbol{\phi})$. Following Liu 
and Sabatti (2000), it can be shown that the Jacobian of this group transformation is $J_{\zeta}=\zeta^{p+G+(G-1) \times q}$ and the Haar measure is $H(d \zeta)=d \zeta / \zeta$. Then the distribution of $\zeta$ is

$$
\begin{aligned}
& \quad \pi\left(\zeta \mid \boldsymbol{\beta}, \boldsymbol{\theta}, \boldsymbol{\phi}, \boldsymbol{\lambda}, \boldsymbol{g}, D_{\text {obs }}\right) \\
& =\prod_{i=1}^{n} \exp \left(\nu_{i}\left\{\log \zeta+\zeta \boldsymbol{x}_{i}^{\prime} \boldsymbol{\beta}-\exp \left(\zeta \boldsymbol{x}_{i}^{\prime} \boldsymbol{\beta}\right) H_{0}^{*}\left(y_{i}\right)\right\}-\zeta \theta_{g_{i}}\left[1-\exp \left\{-\exp \left(\zeta \boldsymbol{x}_{i}^{\prime} \boldsymbol{\beta}\right) H_{0}^{*}\left(y_{i}\right)\right\}\right]\right) \\
& \quad \times \exp \left(\sum_{i=1}^{n}\left[\zeta \boldsymbol{z}_{i}^{\prime} \boldsymbol{\phi}_{g_{i}}-\log \left\{\sum_{l=1}^{G} \exp \left(\zeta \boldsymbol{z}_{i}^{\prime} \boldsymbol{\phi}_{l}\right)\right\}\right]\right) \\
& \quad \times \exp \left(-\frac{\zeta^{2} \boldsymbol{\beta}^{\prime} \boldsymbol{\beta}}{2 c_{01}}\right) \times \prod_{l=1}^{G-1} \exp \left(-\frac{\zeta^{2} \boldsymbol{\phi}_{l}^{\prime} \boldsymbol{\phi}_{l}}{2 c_{02}}\right) \times \prod_{l=1}^{G} \zeta^{a_{l}-1} \exp \left(-\zeta b_{l} \theta_{l}\right) \times \zeta^{p+G+(G-1) \times q-1} .
\end{aligned}
$$

We use the Metropolis-Hastings algorithm to sample $\zeta$ from $\pi\left(\zeta \mid \boldsymbol{\beta}, \boldsymbol{\theta}, \boldsymbol{\phi}, \boldsymbol{\lambda}, \boldsymbol{g}, D_{\text {obs }}\right)$. After we obtain a new $\zeta$, we then adjust $(\boldsymbol{\beta}, \boldsymbol{\theta}, \boldsymbol{\phi})$ by

$$
\boldsymbol{\beta} \leftarrow \zeta \boldsymbol{\beta}, \boldsymbol{\theta} \leftarrow \zeta \boldsymbol{\theta} \text {, and } \boldsymbol{\phi} \leftarrow \zeta \phi
$$

Next, we consider a random $G$. In this case, we develop the reversible jump MCMC (RJMCMC) algorithm based on Lopes and West (2004) and Green (1995).

Let $\boldsymbol{\zeta}=(\boldsymbol{\beta}, \boldsymbol{\theta}, \boldsymbol{\phi}, \boldsymbol{\lambda}, \boldsymbol{N}, \boldsymbol{g})$ denote the collection of parameters except for $G$. Then the dimension of parameters $(\boldsymbol{g}, \boldsymbol{\beta}, \boldsymbol{\lambda}, \boldsymbol{N})$ does not change for different $G$ 's, but the value of $\boldsymbol{g}$ changes with $G$. Let $\boldsymbol{\zeta}^{\prime}$ denote the collection of parameters for the candidate model $G^{\prime}$. For model $G$, the proposal distributions are given as follows:

$$
q_{G}(\boldsymbol{\theta})=\prod_{l=1}^{G} \theta_{l}^{a-1} \exp \left(-a \xi_{l} \theta_{l}\right) \text { and } q_{G}(\boldsymbol{\phi})=\prod_{l=1}^{G-1} N\left(\boldsymbol{\phi}_{l} ; \boldsymbol{b}_{l}, d_{l} B\right)
$$

where $\xi_{l}$ is the posterior mean of $\theta_{l}, \boldsymbol{b}_{l}$ and $B$ are the posterior mean and the variancecovariance matrix of $\phi_{l}$ under model $G$, and $a$ and $d_{l}$ are the prespecified hyperparameters. We further let $p(G)$ be the prior of $G$ with the support $\{1, \ldots, G \max \}$. Then, the RJMCMC algorithm operates as follows.

Step 0. Set the starting values of $G$ and $\zeta$. 
Step 1. Generate a candidate value $G^{\prime}$ from a proposal distribution defined by prespecified transition probabilities $P\left(G^{\prime} \mid G\right)=\operatorname{TR}\left(G \rightarrow G^{\prime}\right)$. Given $G^{\prime}$ and $(\boldsymbol{\beta}, \boldsymbol{\lambda}, \boldsymbol{N})$, generate the parameters $\left(\boldsymbol{\theta}^{\prime}, \boldsymbol{\phi}^{\prime}\right)$ from the distribution $q_{G^{\prime}}(\boldsymbol{\theta})$ and $q_{G^{\prime}}(\boldsymbol{\phi})$ in (S.5), and generate the value of parameter $\boldsymbol{g}^{\prime}$ from the conditional distribution based on $\left(\boldsymbol{\theta}^{\prime}, \boldsymbol{\phi}^{\prime}, \boldsymbol{\beta}, \boldsymbol{\lambda}, \boldsymbol{N}\right)$. Set $\boldsymbol{\zeta}^{\prime}=\left(\boldsymbol{\theta}^{\prime}, \boldsymbol{\phi}^{\prime}, \boldsymbol{g}^{\prime}, \boldsymbol{\beta}, \boldsymbol{\lambda}, \boldsymbol{N}\right)$.

Step 2. Compute an acceptance ratio

$$
\alpha=\min \left\{1, \frac{L\left(\boldsymbol{\zeta}^{\prime} \mid D_{o b s}, G^{\prime}\right) \pi\left(\boldsymbol{\zeta}^{\prime} \mid G^{\prime}\right) \pi\left(G^{\prime}\right) q_{G}(\boldsymbol{\theta}) q_{G}(\boldsymbol{\phi}) \mathrm{TR}\left(G^{\prime} \rightarrow G\right)}{L\left(\boldsymbol{\zeta} \mid D_{o b s}, G\right) \pi(\boldsymbol{\zeta} \mid G) \pi(G) q_{G^{\prime}}\left(\boldsymbol{\theta}^{\prime}\right) q_{G^{\prime}}\left(\boldsymbol{\phi}^{\prime}\right) \operatorname{TR}\left(G \rightarrow G^{\prime}\right)}\right\},
$$

where $L\left(\boldsymbol{\zeta} \mid D_{o b s}, G\right)=L\left(\boldsymbol{\theta}, \boldsymbol{\phi}, \boldsymbol{g}, \boldsymbol{\beta}, \boldsymbol{\lambda} \mid D_{o b s}, G\right)$ is the likelihood function under model $G$ given in Equation (10), $\pi(\boldsymbol{\zeta} \mid G)$ is the prior distribution for the parameters within the model $G$, and $\pi(G)$ is the prior distribution for dimension $G$. Generate $u \sim$ $U(0,1)$ and then set

$$
G= \begin{cases}G^{\prime} & \text { if } u \leq \alpha \\ G & \text { otherwise. }\end{cases}
$$

Step 3. Run one step of MCMC sampling to produce a new value of $\boldsymbol{\zeta}$ under model $G$.

Step 4. Repeat Steps 1 - 3 until practical convergence is judged to have been achieved.

\section{Additional References}

Gilks, W. R. and Wild, P. (1992). Adaptive rejection sampling for Gibbs sampling. Applied Statistics, 41, 337-348.

Hastings, W. K. (1970). Monte Carlo sampling methods using Markov chains and their applications. Biometrika, 57, 97-109.

Liu, J. S. (1994). The collapsed Gibbs sampler in Bayesian computations with applications to a gene regulation problem. Journal of the American Statistical Association, 89, 958-66. 
Liu, J. S. and Sabatti, C. (2000). Generalised Gibbs sampler and multigrid Monte Carlo for Bayesian computation. Biometrika, 87, 353-69. 